\title{
Orson Welles et le remodelage du texte
} shakespearien

\section{François Thomas}

\section{(2) OpenEdition}

\section{Journals}

Édition électronique

URL : http://journals.openedition.org/shakespeare/290

DOI : 10.4000/shakespeare.290

ISSN : 2271-6424

Éditeur

Société Française Shakespeare

Édition imprimée

Date de publication : 1 novembre 1998

Pagination : 171-182

ISBN : 2-84269-230-6

\section{Référence électronique}

François Thomas, "Orson Welles et le remodelage du texte shakespearien », Actes des congrès de la Société française Shakespeare [En ligne], 16 | 1998, mis en ligne le 01 novembre 2007, consulté le 30 avril 2019. URL : http://journals.openedition.org/shakespeare/290 ; DOI : 10.4000/shakespeare.290 


\section{S H A K E S P E A R E \\ \& $\quad$ L E $\quad$ C I N É M A}

Société Française Shakespeare

Actes du Congrès de 1998

米 $*$ *

Textes réunis et présentés par

Patricia DORVAL

publiés sous la direction de

Jean-Marie MAGUIN 
Site web : <http : //alor.univ-montp3.fr/serinf/SFS/> Liste de diffusion : <sfs-1@smrl.univ-montp3.fr >

Tous droits de traduction, de reproduction et d'adaptation réservés pour tous les pays.

(C) 1998. Société Française Shakespeare,

École Normale Supérieure, 45 rue d'Ulm. 75005 Paris.

ISBN 2-84269-230-6 


\section{ORS ON WELLES ET LE REMODELAGE D U TEX TE S HA K ES P E A R I E N}

Je m'attacherai ici à la question de l'adaptation de la façon la plus terre à terre qui soit, celle du traitement du texte même : Orson Welles est confronté, dans le cas par exemple de Macbeth, à 2162 lignes de texte, qu'en fait-il ?

Les remarques qui suivent seront fondées pour l'essentiel sur les trois longs métrages shakespeariens du cinéaste : Macheth (1948), Othello (19491952) et Falstaff (1966), ce dernier inspiré des deux parties d'Henry IV auxquelles s'ajoutent des fragments de Richard II, Henry $V$ et Les joyeuses commères de Windsor. Mais, sans parler d'un Marchand de Venise inédit tourné en 1969 pour la télévision, l'œuvre théâtrale et radiophonique de Welles est clle aussi fortement placée sous le sceau shakespearien. Sur les planches, outre une impressionnante liste de mises en scène amateur et ses premières interprétations professionnelles pour le compte d'autrui, Welles a monté sept spectacles shakespeariens. Parmi eux, cinq sont en rapport direct avec ses films. Après son Macbeth "vaudou» de 1936 interprété par une troupe entièrement composée de Noirs, sa seconde mise en scène de la pièce, en 1947, était une sorte de répétition générale du film réalisé dans la foulée. Son Othello de 1951 a pu jouer in extremis sur certains détails de la finition de l'adaptation cinématographique déjà tournée. Et deux spectacles condensant plusicurs pièces avaient préfiguré Falstaff: Five Kings en 1939, dont la première partie, seule représentée, couvrait les règnes d'Henry IV et Henry V, et Chimes at Midnight en 1960, au texte plus proche de celui du film qui lui doit son titre original. À la radio, Welles a notamment donné en 1938 une adaptation de son Jules César scénique créé l'année précédente. Il a également enregistré en 78 tours quatre pièces, parmi lesquelles Macbeth, dont il a 
cosigné une édition scolaire illustrée, annotée et préfacée par lui. Or c'est son expérience de la scène et des ondes qui a formé Welles à l'art de l'adaptation, et en particulier de l'adaptation shakespearienne. Les mêmes procédés, peu ou prou, sont repérables dans son ceuvre théâtrale et radiophonique dès les annécs 30 avant de s'étendre à la totalité de ses entreprises shakespeariennes.

Welles soumet la matière brute qu'est pour lui le texte du dramaturge à toutes sortes de manipulations, à l'échelle aussi bien du vers ou de la réplique que de la structure de la scène ou de la pièce. Il ne se contente pas de couper dans le texte par grands blocs, mais se livre à une chirurgic de tous les instants. Des trois films, Macheth, où la stylisation rugueuse de la diction (à commencer par l'accent écossais adopté par les comédiens) l'cmporte sur l'individualisation vocale des personnages, est celui qui par certains aspects manifeste le plus son origine théâtrale. Les plans longs y vont de pair avec un texte plus continu, plus proche de l'original. Avec Othello, au montage morcelé s'il en est, commence véritablement la recomposition d'un texte source aux éléments disséminés. Falstaff, dont l'intrigue est la plus refondue et dont le dialogue recherche davantage une impression de naturel, est l'(xuvre qui s'octroie les coudées les plus franches.

Une dernière précision: Welles a signé deux montages différents de Macbeth et d'Othello. À la demande de la société de production, il a remonté le premier de ces films après l'échec public et critique d'une sortie «de rodage» limitée à un petit nombre de villes. Et il a atténué certains traits stylistiques hétérodoxes du sccond en 1955, trois ans après sa distribution dans les pays francophones, pour sa diffusion aux États-Unis et en Grande-Bretagne. Les différences entre ces versions, pour importantes qu'elles soient, débordent le cadre de ces pages '. Qu'il suffise de savoir que dans les deux cas je me réfêre à la première version.

\section{La proportion conservée du texte}

L'exigence suprême, pour Welles, est celle de la brièveté, de la concision, garantes du dynamisme recherché. Ses représentations théâtrales ne duraient souvent qu'une heure et demie, ses dramatiques radiophoniques des années trente tenaient en une heure. Les films, eux, font 1 h48 (Macbeth), lh34 (Othello) et 1 h55 (Falstaff).

Welles a conservé près de la moitié du Macbeth de Shakespeare, un tiers d'Othello, et, dans Falstaff, un quart des deux Henry $I V$, pour m'en tenir aux pièces qui fournissent à ce dernier film l'essentiel de son texte. Comparés aux autres réalisations du cinéaste, Macbeth et Othello se signalent par leur faible densité en dialogue. Le premier a toutefois un dialogue toujours compact, continu, alors que dans le second le dialogue, distendu, ne forme pas le cceur des séquences. Même si la chose est moins vraie dans Falstaff, les films shakespeariens de Welles sont paradoxalement ceux qui, en termes de quantité, 
reposent le moins sur le dialogue.

\section{La proportion relative de prose et de poésie dramatique}

Dans Macbeth et Othello, malgré l'ampleur des coupes, Welles est ćtonnamment retombé sur la répartition entre prose et poésie dramatique qui figurait dans les pièces, à la seconde décimale près pour le premier et à $0,5 \%$ près pour le second: Macbeth compte $6,75 \%$ de lignes de prose et Othello $22 \%{ }^{2}$. Dans Falstaff, il a au contraire métamorphosé l'aspect d'ensemble des deux Henry $I V$, qui comportent respectivement $19,3 \%$ et $38,6 \%$ de lignes de prose : il a, en moyenne, presque doublé cette proportion pour arriver à $6.7,7 \%$. Welles, en recentrant l'intrigue autour des relations du prince $\mathrm{Hal}$ avec Falstaff et Henry IV, et dans une moindre mesure avec Hotspur, a supprimé ou réduit à la portion congrue les personnages secondaires de la cour. Il a ainsi accentué l'opposition entre la poésic dramatique au château royal et la prose à l'auberge, domaine de Falstaff. Le prince, quant à lui, alterne cntre les deux types d'élocution selon la compagnie dans laquelle il se Irouve. Ce contraste, pour sortir un instant de la seule question de l'établissement du dialogue, s'étend à toute l'esthétique vocale du film: personnages statiques dans un cas, virevoltants dans l'autre, ce qui rejaillit sur la diction; rigueur verbale d'Henry IV et de ses courtisans, grognements, sifllements, claquements de langue, gloussements de Falstaff et de ses compagnons ; répliques menées à leur terme d'Henry IV, interruptions et chevauchements (j'y reviendrai) de Falstaff et consorts ; paroles mesurées résonnant en écho dans les pièces vides et froides du château de pierre, chaleur el liberté sonores de l'auberge de bois.

\section{La Iongueur moyenne des répliques en vers}

Welles, par rapport aux pièces, a réduit cette longueur moyenne d'environ $40 \%$ pour les deux premiers films et d'environ $45 \%$ pour le troisième ${ }^{3}$ : elle est de 2,7 vers pour Macbeth, de 2,2 vers pour Othello et de 3,2 vers pour Falstaff. Si ce dernier film, qui comporte de très loin le plus de prose, est pourtant celui aux répliques versifiées les plus étendues, c'est précisément que Welles y oppose souvent de longues tirades en vers solennels à la cour à des répliques en prose nettement plus brèves (leur longueur moyenne est de 1,7 ligne) à l'auberge, échangées à la vitesse de l'éclair.

Cortes, Welles a conservé, notamment pour Henry IV, des tirades d'une certaine ampleur, mais elles ne dépassent pas vingt-huit vers (contre soixantetrois dans les pièces), à l'exception du discours d'Othello au Sénat qui en compte cinquante-quatre. Même ces répliques-là sont le plus souvent écourtées, hormis justement le discours au Sénat qui, s'il perd quelques vers, est la combinaison en un seul bloc des deux grandes tirades distinctes de la pièce (1.3.76-94 et 127-69). 


\section{Le démembrement de répliques dont les éléments sont répartis sur plusieurs personnages}

Ce démembrement, systématique chez Welles, contribue lui aussi à accélérer le dialogue. Le cinéaste orchestre des passages d'échanges rapides de répliques brèves, tendant vers la stichomythie, quitte à les créer de toutes pièces. Donnons-en deux excmples.

Dans Macbeth, Welles morcèle de façon privilégiée les répliques des sorcières. Dans le prégénérique où le trio fatidique complote contre Macheth absent en empruntant son texte aux scènes I.1 et IV.1 de la pièce, Welles, après que les sorcières ont prononcé en chœur les deux premiers vers, répartit entre elles trois les quinze suivants qu'il découpe au point d'en tirer vingtdeux répliques. Ou bien, alors que Macbeth et Banquo s'apprêtent à quitter la lande où ils ont rencontré les sorcières, les deux vers de l'aparté final de cellesci (puisés dans le texte d'Hécate, III.5.30-1) sont décomposés en cinq répliques : «He shall spurn fate...», «... scorn death...», «... and bear / His hopes 'bove wisdom...», «... grace...», «... and fear».

Dans Falstaff, lorsque le gras chevalier, avant de se mettre en route pour la guerre contre les rebelles, est pris à partie par deux de ses ennemis de la cour, Welles scinde une réplique de prose du lord grand juge (2 Henry IV, I.2.180-7) en dix répliques confiées alternativement à leur titulaire initial ct à un personnage, l'évêque, inventé pour la circonstance :

Shakespeare

Ch. Just. Do you set down your name in the scroll of youth, that are written down old with all the characters of age? Have you not a moist eye, a dry hand, a yellow cheek, a white beard, a decreasing leg, an increasing belly? Is not your voice broken, your wind short, your chin double, your wit single, and every part about you blasted with antiquity? And will you yet call yourself young?
Welles

Bish. Do you set down your name in the scroll of youth?

Ch. Just. You that are written down old with all the characters of age?

Bish. Have you not a moist eye, a dry hand, a yellow cheek...

Ch. Just. ... a white beard...

Bish. ... a decreasing leg...

Ch. Just. ... an increasing belly?

Bish. Is not your voice broken, your wind short...

Ch. Just. ... your chin double...

Bish. ... your wit single...

Ch. Just. ... and every part about you blasted with antiquity? And will you yet call yourself young? 


\section{Le chevauchement du dialogue}

Wclles accentue ce goût de l'accélération en recourant au chevauchement du dialogue, technique apparue sur la scène américaine à la fin des années vingt mais que, avec son Jules César de 1937, il aurait été le premier à mettre en cxuvre dans un spectacle shakespearien. Lorsqu'il ne fait pas s'enchaîner rapidement les répliques, il les fait mordre les unes sur les autres. Ce style de jeu relève avant tout de la direction d'acteurs, mais, envisagé dès le stade de l'adaptation, il commande aussi la conception de celle-ci. Les remaniements du texte créent souvent des situations de chevauchement. Pour n'en donner qu'un exemple, lorsque Hal et Poins, après le tour qu'ils ont joué incognito à Falstaff dans la forêt de Gadshill, le font s'enferrer dans les histoires à dormir debout pour nier sa couardise, Welles réduit à quelques mots une réplique du bravache (I Henry IV, II.4.165-7) :

\section{Shakespeare}

Fal. $[\ldots]$ Let them speak. If they speak more or less than truth, they are villains and the sons of darkness.

Prince. Speak, sirs, how was it?

Et, le prince attaquant sa réplique avant que Falstaff ait fini la sienne, les dcux «spcak» prononcés par l'un et l'autre personnage sont exactement simultanés, les deux répliques s'unissent en une seule coulée vocale.

Welles, tablant sur le fait que le spectateur n'y verra que du feu, va jusqu'à faire répondre un personnage avant que son interlocuteur ait prononcé les mots justifiant la riposte. Certes, dans ses films shakespeariens, la grande majorité des chevauchements sont des empiétements modestes mordant tout au plus sur une syllabe ou deux, et il s'y livre sensiblement moins qu'il ne l'avait fait sur la scène dans Five Kings, sans parler de ses films à sujet contemporain dont beaucoup relèvent à cet égard du tour de force. La proportion de répliques participant à ce jeu, tout à fait inhabituelle, n'en reste pas moins de 13\% dans Macbeth, 23\% dans Othello et 31\% dans Falstaff, film où le chevauchement, appliqué bien davantage à la prose qu'aux vers, vise à l'impression de naturel.

\section{L'attribution de répliques à un autre personnage}

Ces redistributions ne se limitent pas au repêchage de répliques d'un comparse supprimé par l'adaptation, mais sont dues aussi à la volonté de 
varier les interlocutcurs ou au contraire d'en réduire le nombre, ou à des retouches délibérécs de la psychologie des personnages. Un personnage peut ainsi répondre à son interlocuteur avec le texte même de celui-ci. Welles a pratiqué une cinquantaine de ces licences dans Macbeth, une trentaine seulement dans Othello et quelque quatre-vingt-dix dans Falstaff. Encore ne compté-je pas dans Macbeth la création d'un rôle composite, devenu même le quatrième du film en nombre de répliques : un prêtre chargé d'illustrer le conflit entre paganisme et christianisme sur lequel Welles a fondé son adaptation. Le dialogue du saint homme amalgame des répliques empruntées à cinq personnages secondaires, mais aussi à Banquo et Macbeth.

Un cas particulier est celui de la lettre que Macbeth, après les premières prédictions des sorcières, adresse à son épouse qui la lit à voix haute chez Shakespeare (1.5). Dans le film, Macbeth qui dicte son message au prêtre étant relié en fondu enchainé à lady Macbeth qui en prend connaissance, la fin de la lettre est lue simultanément par l'expéditeur et par la destinataire : la voix intérieure de lady Macbeth, bouche fermée, se superpose à celle de son mari pour les derniers mots, après quoi elle se réjouit des nouvelles reçucs.

\section{La raréfaction des soliloques}

Macbeth est le seul des trois films à conserver un nombre important de soliloques, réservés au couple criminel. En dehors de simples apartés, du soliloque de lady Macbeth évoqué à l'instant et de la crise de somnambulisme, Macbeth prononce huit soliloques en voix intérieure ou, pour «Tomorrow, tomorrow, and tomorrow...» (V.5), sur des images de brumes en mouvement qui reflètent le chaos, l'indécision évoqués par le texte. Le cinéaste accuse ainsi l'isolement de ce héros qui ne peut se confier qu'à lui-même et se désole de ne plus pouvoir compter sur des «troupes d'amis», et accroît sa suprématic sur son épouse en termes de dramaturgie.

Dans Othello, Welles renverse l'équilibre entre Iago et le More de Venise. Abolissant la distinction entre ce que le scélérat donne à voir à ses dupes et au public (d'où la pleine opacité de sa «méchanceté sans mobile»), il supprime ou convertit en dialogue chacun des huit soliloques par le biais desquels il expose dans la pièce ses intentions au spectateur. Il préserve en revanche un des trois soliloques d'Othello, qu'il scinde en deux tirades distinctes, et en forge un nouveau à partir d'une réplique adressée à lago. Dans Falstaff, le prince conserve ses deux soliloques et le roi son invocation au sommeil, mais Welles, à deux lignes près, veille à ce que le cabotin Falstaff, détenteur de sept soliloques chez Shakespeare, s'adresse toujours à un public.

\section{La substitution de mots}

Welles procède à une vingtaine de ces substitutions dans Macbeth, à une quarantaine dans Othello et à quelque cent vingt dans Falstaff. Les libertés 
plus grandes de ce dernier film s'expliquent notamment par la prédominance de la prose et par le tour plus quotidien de la diction et du style d'ensemble. Parmi ces modifications, beaucoup sont négligeables : «that» pour «which» ou pour «who», «should» pour «would», «shall» pour «will», etc. Pour des raisons de cohérence impliquées par la restructuration du texte, Welles a été conduit à remplacer un pronom par un nom, un singulier par un pluriel ou un temps verbal par un autre. Les substitutions les plus voyantes sont souvent elles aussi utilitaires: Iago n'accuse plus Cassio, glabre dans le film, de s'éponger la barbe mais le visage avec le mouchoir de Desdémone (III.3.436), ou bicn Macbeth, imputant le meurtre du roi Duncan à Malcolm et Macduff (plutôt qu'à Malcolm et Donalbain comme dans la pièce), n'emploie plus le mot de «parricide» mais celui de «murders» (III.1.31). Il s'agit enfin d'assurer la compréhension immédiate quand "guardage» est remplacé par «father» (Othello, 1.2.69) ou «caitiff» par "creature» (même pièce, IV.1.108). Les modifications de Macbeth sont très en retrait de celles réclamées par la société de production qui, à la lecture du premier scénario shakespearien hollywoodien depuis plus de dix ans, espérait voir disparaître des mots obscurs tels que «incarnadine» ou même «augurs».

\section{L'ajout de mots ou de répliques}

Ces ajouts, toujours discrets voire indétectables, sont au nombre d'une trentaine dans Macbeth, de quelque soixante-dix dans Othello et de quelque cent trente dans Falstaff. Ils consistent dans une large mesure en apostrophes telles que «My lord !», en simples redoublements d'expressions figurant dans la pièce ou en interrogatives destinées à relancer le dialogue, parfois pour raccorder avec plus de liant deux passages éloignés chez Shakespeare. Quelques-uns de ces ajouts sont moins innocents. Lorsque Falstaff demande à l'assesseur du juge Shallow s'il se nomme bien Surecard (2 Henry IV, III.2.86), Welles fait s'écrier brutalement à celui-ci : "Silence !», et le retour à la réplique suivante de la pièce en change le sens : le juge ne répond plus à la place de son collègue, mais il prétend, embarrassé, que son «cousin Silence» vient de se présenter. Ou bien, lorsque Henry $V$, avant de s'embarquer pour la France, demande que l'on relâche l' «homme arrêté hier» qui demeure anonyme chez Shakespeare (Henry $V$, II.2.40), l'injection de la question du lord grand juge : «Falstaff ?» suffit à adoucir, après la séquence du bannissement, la sévérité du nouveau roi envers son ancien compagnon emprisonné.

\section{Les modifications du mètre}

Ces modifications sont inévitables dès lors que les décasyllabes iambiques sont tronqués. La régularité du vers disparaît ainsi pour une bonne part, quoique sensiblement moins dans Macbeth. Lorsqu'il substitue un mot à 
un autre, Welles veille toutefois en règle générale à respecter le mètre. Il accole aussi très fréquemment deux hémistiches. La critique anglaise l'a surtout attaqué pour avoir amendé la fin du premier vers du soliloque d'Othello «It is the cause, it is the cause, my soul» (V.2.1) en «O, my soul», altération destinéc, sclon le monteur de la version anglo-saxonne du fílm ${ }^{4}$ (la seule où elle figure), à accorder le texte au rythme visuel.

Il ne faut nullement en déduire, si l'on m'autorise ici une parenthèse, que Welles cherche à déprécicr ou à effacer le vers. Au contraire, dès son Macbeth théâtral de 1936, il fait du vers l'unité de base d'une mise en valeur invisible du texte par le bruit et la musique, lesquels sont placés de façon rigourcuse en fonction de ses contours. Parmi les trois films, Macbeth surtout, le plus versifié, sollicitait un tel habillage sonore. Le tonnerre, le vent, des timbales solo voire l'orchestre soulignent un nombre donné de vers, soutiennent la voix dont ils renforcent le côté hérö̈que ou blafard selon le contexte. Des bruits récurrents plus brefs (coups de bélier, tocsin...) ponctuent les fïns de vers ou les césures. Ou encore l'arrêt abrupt d'un bruit ou d'une musique détache le dernier vers d'une tirade. Le bruit et la percussion mettent ainsi à nu les divisions du dialogue de maintes séquences, délimitant par exemple six des huit soliloques de Macheth. Othello et Falstaff prolongent ce style de façon plus épisodique mais souvent tout aussi marquante.

\section{Le changement de l'ordre des scènes}

À l'aune des deux autres films, Macbeth, où la chronologie d'ensemble de la pièce est grosso modo respectée, offre peu de telles interversions. Dans Othello, si le début et la fin ne présentent guère de changements majcurs (hormis le prégénérique muet dont l'action est postérieure à celle de la pièce : cortège funèbre des époux défunts et d'Emilia, châtiment du traître), l'évolution des manœuvres de Iago et des réactions d'Othello est bouleverséc. D'un bout à l'autre de Falstaff, Welles s'est livré à une restructuration complète des pièces sources. Le film s'ouvre lui aussi par un prégénćrique, dialogué celui-là, où Falstaff et Shallow usés empruntent leur texte nostalgique à 2 Henry IV (III.2). Welles divise l'œuvre en deux partics au net changement de climat et dont la trame générale correspond schématiquement à celle des deux pièces, mais il ne cesse de transposer des éléments d'une partie à l'autre. Notamment, il place avant la bataille de Shrewsbury (I Henry IV, V.3-5) la scène de recrutement chez le juge Shallow (mélange de 2 Henry $I V$, III.2 et V.1) et autres préparatifs militaires qui précèdent chez Shakespeare le conflit résolu devant la forêt de Gaultree, épisode supprimé du film. Ou bien il remonte en arrière dans les pièces pour créer l'unité thématique des trois séquences qui trahissent tour à tour l'humeur morose du roi (l'invocation au sommeil citée, 2 Henry IV, III.1) puis, dès les premiers vers des séquences suivantes, du prince («Before God, I am exceeding weary...», II.2) et de 
Falstaff («'Sblood, I'm as melancholy as a gib cat, or a lugged bear», l Henry IV, I.2).

Opérant ces remaniements, Welles accorde un soin particulier aux effets de transition ou de contraste que permettent les ouvertures et fermetures de séquences. Les mots de Hotspur disant son mépris du prince Hal qu'il aimerait faire empoisonner avec un pot de bière (I Henry IV, I.3.225-31) sont postéricurs chez le dramaturge à notre première rencontre avec le prince, qui a cu licu en 1.2. Welles, lui, s'en sert pour ménager la transition avec la séquence suivante, dont le premier plan montre précisément Hal, avec qui nous faisons seulement connaissance, un pot de bière aux lèvres. Ou bien encore, quand Hal et Poins commentent avec exultation leur farce de la forêt de Gadshill ( $I$ Henry IV, II.2), l'entrain s'efface net devant la froideur de la grande porte du palais qui s'ouvre avec fracas et la question véhémente que le roi lance à la cantonade : «Can no man tell me of my unthrifty son ?». Cette entrée en matière saisissante est empruntée à Richard $I, \mathrm{~V} .1$, où Henry Bolingbroke n'est pas encore roi.

Dans Macbeth, Welles a placé à la toute fin, apaisée après la chute du tyran, les mots «Peace! The charm's wound up» (1.3.36) confiés à une des sorcières. Dans son contexte originel, l'expression signific que le sortilège est prêt, mais sa position terminale lui fait dire que le sortilège est parvenu à son tcrme («to wind up» a aujourd'hui les deux sens). La critique américaine ayant protesté contre ce détournement lors de la sortie «de rodage» du film, les derniers plans de la version remontée sont muets.

\section{Le remodelage de l'ordre des répliques à l'intérieur d'une séquence}

Ce remodelage est constant dans les trois films, au point de figurer dans presque toutes les séquences de quelque longueur. Celle d'Othello équivalant à V.2 (le meurtre de Desdémone et la fin de la pièce) contient par exemple une trentaine de va-et-vient dans la scène shakespearienne. Donnons un exemple plus réduit de ce type d'allers-retours avec l'arrivée d'Othello à Chypre (II.1) : se succèdent les lignes 1-2, 88-9, 17-9, 174, 20-2, 196, 164-6, 177-80, 176b, 176a, 200-1 et 206, cela sans tenir compte de brèves répliques forgées pour la circonstance ni de deux vers importés d'une scène ultérieure. Mais l'aspect purzle de séquences plus longues est beaucoup plus prononcé.

Par le biais de ces interversions aussi, Welles s'ingénie à inventer des déhuts de séquence percutants in medias res. Après le générique de Falstaff, il attaque ainsi avec les vers $84-5$ de 1 Henry $I V$, I.3 qui traduisent la fureur du roi : «Shall our coffers then / Be emptied to redeem a traitor home?», puis, quatre répliques plus loin, il revient aux premiers vers de la scène. 
Le mélange, dans une séquence, d'éléments empruntés à plusieurs scènes

Cet autre procédé généralisé va fréquemment jusqu'au brassage complet des scènes sources. Dans Macbeth, certes, les répliques proviennent davantage en continu d'une même source (certaines séquences y ont même une source unique), et Welles amalgame des scènes en règle générale peu éloignćes les unes des autres dans la pièce. Il en va tout autrement dans Othello et Falstaff. Le dialogue de la longue séquence où nous faisons connaissance avec Hal en même temps qu'avec Falstaff, Poins et autres compères est par exemple formé d'un va-et-vient entre trois scènes de $l$ Henry $I V$ puisées chacune dans un acte différent et d'une scène de 2 Henry $I V$, sans parler d'un court emprunt chacun à Henry $V$ et aux Joyeuses commères de Windsor. Welles forge aussi par ce biais des conversations parallèles tenues par deux groupes distincts de personnages. Tandis que Hal, mélancolique à l'heure où son père est rongé par la maladie, confie à Poins se sentir «bien blâmable de profaner ainsi en folies un temps précicux» puis se lance dans un bref aparté, Falstaff, à bonne distance, radote mi-pour la galeric, mi-pour le prince: Welles a pioché le texte de Hal et Poins dans 2 Henry IV, II.2 et II.4, et celui de Falstaff dans 1 Henry IV, I. 2 et II. 2 et 2 Henry IV, V.I.

\section{Les interpolations}

L'aspect composite de Falstaff et de ses prédécesseurs Five Kings et Chimes at Midnight étant leur raison d'être, le terme d' «interpolation» est sans doute impropre à leur égard, encore que l'injection de lambeaux des Joyeuses commères de Windsor au milieu des pièces historiques soit peu orthodoxe. Dans Macbeth, Welles a renforcé la présence du christianisme en plaçant deux prières dans la bouche du prêtre, dont une en latin. L'interpolation est surtout à chercher dans le Jules César théâtral de 1937 : dans la scène avec Cinna le poète (III.3), Welles a donné aux citoyens insurgés après l'oraison funèbre prononcée par Marc Antoine de brèves répliques empruntées aux insurgés de Coriolan (I.1) ; et la chanson de Lucius réclamée dans IV.3 est celle chantée pour la reine Catherine dans Henry VIII (III.1).

\section{L'adjonction de narrations off tirées des sources de Shakespeare}

Welles a étrenné à la radio ce retour aux sources des pièces. Dans sa mise en ondes de Jules César citée, retrouvant d'une autre façon l'aspect contemporain de son spectacle en costumes modernes, il a ajouté une narration tirée de Plutarque et lue par un des commentateurs d'actualités vedettes de son temps. Dans Five Kings, il a étoffé le rôle du Chœur (cclui-ci, dans les pièces utilisées, ne figure que dans Henry $V$ ) en lui offrant du texte 
emprunté, avec divers remaniements, aux Chroniques de Holinshed. Le Chœur assurait ainsi la continuité, altérée par la restructuration des pièces, de ces «morceaux choisis» et permettait de s'y retrouver dans la généalogie royale anglaise. Dans Chimes at Midnight et Falstaff, Welles n'a même utilisé que Holinshed. Dans la version anglo-saxonne d'Othello, il a remplacé le générique parlé de son premier montage, parfois jugé déroutant, ainsi que les discussions des sénateurs vénitiens sur la guerre avec Chypre par une narration off explicative tirée de la nouvelle de Cinthio ${ }^{5}$.

\section{Pour conclure}

Toutes les libertés que nous venons de voir peuvent paraître énormes si l'on considère la déférence observée par la majeure partie des cinéastes anglosaxons à l'égard du texte shakespearien. Pourtant, Welles, dont la plupart des films sont des adaptations, fait preuve avec le dramaturge anglais du respect à ses ycux maximal de la lettre du texte. Qu'on songe par exemple au scénario non tourné d'Au cour des ténèbres (1939) ou à Une histoire immortelle (1968) : s'attaquant, avec Conrad et Isak Dinesen, à deux de ses écrivains de prédilection dont il admirait la pureté de l'anglais, il se livre à des modifications autrement profondes, notamment en termes de substitutions et d'ajouts. Et lorsqu'il adapte des romans policiers de second rayon avec $L a$ dame de Shanghai (1947) ou La soif du mal (1958), il se permet tout, au point de ne garder dans le premier de ces films qu'une dizaine de répliques à peu près intactes. Au théâtre, seul Marlowe, dont il a monté La tragique histoire du docteur Faust en 1937, a bénéficié du même traitement que Shakespeare. Et quand G.B. Shaw, l'année suivante, a exigé que l'on ne modilie ni ne retranche un seul mot de La maison des caurs brisés, Welles en a perdu une grande part de sa capacité d'invention. Adapter, pour lui, c'est remodeler, reconstruire, bouleverser - créer une æuvre nouvelle. Cette recomposition est plus aisée à accomplir avec un roman ou un récit qu'avec une ceuvre dramatique. Dans le second cas, outre l'immersion de toute une vie dans l'cxuvre de Shakespeare, c'est la versification qui interdit au cinéaste d'opérer des altérations aussi radicales que celles auxquelles il est accoutumé. Les mots qu'il a conservés sont presque sacrés, mais leur ordre, leur attribution voire leur sens ne le sont pas le moins du monde: Shakespeare propose, Welles dispose. 


\section{N O T E S}

1 J'en propose une recension dans «Les mésaventures d'une bande sonore: les deux Macbeth d'Orson Welles», Cinémathèque, n 6, automne 1994, p. 12540, et dans «La tragédic d'Othello», Positif, n ${ }^{\circ} 424$, juin 1996, p. 70-6. La version d'Othello «restaurée» en 1992, seule distribuée aujourd'hui, est une adultération du second montage de Welles dont on a trafiqué numériquement une grande part des dialogues, compressant ou allongeant les syllabes au mépris de l'intégrité de l'interprétation.

${ }^{2}$ Les chiffres shakespcariens, comme plus loin pour les deux Henry IV. sont donnés par Henri Suhamy, Le vers de Shakespeare, Atelier national reproduction des thèses, université Lille III, 1984, p. 73-4. Pour les films de Welles, je ne prends en compte que le texte issu de Shakespeare, à l'exclusion des répliques ajoutées par le cinćaste (voir plus loin).

3 Je me fonde de nouveau sur les chiffres shakespeariens donnés par H. Suhamy (op. cit., p. 37I), en faisant la moyenne pour les deux Henry IV.

${ }^{4}$ Cf. Frank Brady, Citizen Welles: A Biography of Orson Welles. New York, Charles Scribner's Sons, 1989, p. 444.

${ }^{5}$ Dans la seconde version de Macbeth, il a également enregistré par concession un commentaire inaugural de son cru situant le drame dans la vicille Écosse et en dégageant les thèmes. 